\title{
Increased serum level of early prostate cancer antigen is associated with subsequent cancer risk in men with high-grade prostatic intraepithelial neoplasia
}

\author{
Zhigang Zhao and Guohua Zeng
}

Department of Urology of Minimally Invasive Surgery Center, the First Affiliated Hospital of Guangzhou Medical College, No. 1-3, Kangda Road, Guangzhou 510230, Guangdong Province, People's Republic of China

(Correspondence should be addressed to G Zeng; Email: gzgyzgh @ vip.tom.com)

\begin{abstract}
Early prostate cancer antigen (EPCA) has been recently suggested as a novel biomarker in malignant and premalignant lesions of the prostate. This study was to examine serum expression of EPCA and to further clarify the relationship between initial serum EPCA levels and the presence of subsequent cancer in the individuals with isolated high-grade prostatic intraepithelial neoplasia (HGPIN). An indirect ELISA was used for initial serum EPCA measurement in 112 men with isolated HGPIN, who were enrolled and completed a follow-up of $\geq 5$ years. All patients had a detectable concentration of EPCA in the initial serum, with a mean of $0.64 \pm 0.13$ absorbance at $450 \mathrm{~nm}$. Thirty-three patients had an initial serum EPCA level of $\geq 1.10$, in which 31 cases were subsequently identified as having prostate cancer on follow-up. However, in the remaining 79 cases, serum EPCA levels were all $<1.10$, and none was diagnosed with cancer later. Statistical analysis showed a significantly higher serum ECPA level in isolated HGPIN patients with subsequent cancer than those without cancer $(P<0.001)$. The area under the receiver operating characteristic curves showed that serum EPCA level had better predictive accuracy of cancer onset on follow-up than prostate specific antigen velocity and abnormal digital rectal examination findings. Furthermore, univariate and multivariate Cox regression analyses demonstrated the predictive performance independently by initial serum EPCA $\geq 1.10$ absorbance (relative risk, 3.32; 95\% confidence intervals, 2.62-5.03, $P<0.001$ ). These preliminary findings first show the potential of serum EPCA to serve as a significant predictor for subsequent cancer in isolated HGPIN.
\end{abstract}

Endocrine-Related Cancer (2010) 17 505-512

\section{Introduction}

High-grade prostatic intraepithelial neoplasia (HGPIN) is currently accepted as a risk factor for the delayed progression to prostate cancer (PCa; Dovey et al. 2005, Ayala \& Ro 2007, Chin et al. 2007, Montironi et al. 2007, Godoy \& Taneja 2008). The incidence rate of carcinoma detection in repeat biopsies after a HGPINpositive biopsy ranges from 22 to $60 \%$ (Dovey et al. 2005, Gokden et al. 2005, Joniau et al. 2005, Moore et al. 2005, Freeman 2006, Herawi et al. 2006). To date, no pathologic, molecular, and clinical variables help identify which men with HGPIN are at increased risk for developing PCa (Abdel-Khalek et al. 2004, Roscigno et al. 2004, Singh et al. 2004, Chin et al. 2007, Loeb et al. 2007).

The alterations in the protein components of the nuclear structure have been associated with carcinogenesis in a variety of tissues, including prostate. Thus, nuclear matrix proteins have been initially identified with which it is possible to differentiate between normal prostate and prostatic carcinoma. Early prostate cancer antigen (EPCA), a nuclear matrix protein 
recently discovered by Dhir et al. (2004), was identified as being expressed throughout the prostate of patients with $\mathrm{PCa}$ but not in those without the disease. A recent ELISA for plasma EPCA showed, using a cut-off of 1.7 absorbance at $450 \mathrm{~nm}$, that EPCA had a sensitivity of $92 \%$ (11/12) for patients with PCa, a specificity of $100 \%$ (16/16) for healthy donors, and an overall specificity of $94 \%$ (32/34) for entire donor controls (Paul et al. 2005). Most recently, EPCA-2.22, one of three sequenced epitopes of the EPCA-2 (another nuclear structural protein which is unrelated to EPCA), has been identified as having a $92 \%$ specificity for healthy men and for men with benign prostatic hyperplasia, and a 94\% sensitivity for overall PCa patients using a cut-off value of $30 \mathrm{ng} / \mathrm{ml}$ (0.8 absorbance at $630 \mathrm{~nm}$; Leman et al. 2007). EPCA-2.19, the second epitope of EPCA-2, also has a specificity of $94 \%$ and a sensitivity of $91 \%$ in separating normal men with prostate specific antigen (PSA) $<$ and $>2.5 \mathrm{ng} / \mathrm{ml}$, and men with BPH from those with $\mathrm{PCa}$ at a cut-off of $0.5 \mathrm{ng} / \mathrm{ml}$ and above (Leman et al. 2009). These data have shown the potential of EPCA and EPCA-2 to serve as promising serum biomarkers for the detection of PCa. The positive immunostaining for EPCA protein was noted not only in the benign glands of the negative biopsies from men who, as much as 5 years later, were eventually detected as having prostate carcinoma but also in HGPIN in foci adjacent to PCa (Dhir et al. 2004, Uetsuki et al. 2005). These findings suggest that upregulation of EPCA may be involved in the presumably earlier stage of prostatic carcinogenesis and that EPCA could be used as a potential predictive marker. However, to the best of our knowledge, there are no published reports on serum EPCA expression and the relationship between serum EPCA levels and the clinical outcome in men with a diagnosis of isolated HGPIN. Therefore, we examined the serum levels of EPCA by using an indirect ELISA approach in 112 cases with isolated HGPIN diagnosed on initial biopsies, and we further cleared whether serum EPCA level is a significant predictor of subsequent malignancy onset in those patients during a follow-up period of $\geq 5$ years.

\section{Materials and methods}

\section{Patients}

Between January 2000 and May 2003, 687 men underwent transrectal ultrasound-guided needle biopsy of the prostate at our institution by using a standardized 12-cores biopsy scheme. This scheme was defined by two consecutive sets of sextant biopsies from the peripheral zone on the right and left. One hundred and forty-three $(20.8 \%)$ men were histopathologically diagnosed with HGPIN, and they had no focus of concurrent cancer on initial biopsy, i.e. isolated HGPIN. No patients had a previous biopsy, a previous diagnosis of PCa, and had ever received androgen deprivation therapy, chemotherapy, or radiation therapy. Of those 143 patients with isolated HGPIN, 112 men had one blood draw on the morning of the scheduled day of prostate biopsy after an overnight fast at our department, and the volume of samples was sufficient for the determination of serum EPCA and total PSA levels. Thus, a total of 112 isolated HGPIN patients were enrolled into this study. The blood samples were collected in evacuated tubes. Serum was separated by centrifugation at $1200 \mathrm{~g}$ for $10 \mathrm{~min}$ at room temperature, and then stored immediately at $-80{ }^{\circ} \mathrm{C}$ until analysis. Serum total PSA was measured by RIA (Hybritech Inc., San Diego, CA, USA) in each case. All patients provided a signed study-specific informed consent form prior to having the blood sampled, and the Institutional Review Board approved this study protocol.

\section{Indirect ELISA}

A commercially available anti-EPCA polyclonal antibody (Onconome Inc., Seattle, WA, USA) was used, and an indirect ELISA for EPCA was performed as described recently by Paul et al. (2005). Here, serum sample from each patient was plated in triplicate in 96-well Nunc Immunoplate MaxiSorb plates (Nunc, Wiesbaden, Germany). When the reaction was stopped, the plates were read at $450 \mathrm{~nm}$ on the Labsystems Multiskan Multisoft microplate reader (LabSystems, Helsinki, Finland). The mean of the three measurements for each sample was used for data analyses. In the present study, all the assays were performed by an experienced laboratory expert at our institute ( $\mathrm{G} \mathrm{Z}$ ), who had no knowledge of the case status of the samples.

\section{Follow-up study}

All 112 men with isolated HGPIN were followed continuously for a minimum of 5 years from the date of the initial diagnosis. During this period, all the cases had the first 12-core follow-up biopsy of prostate within 18 months of the diagnosis of HGPIN alone on their initial biopsy. The patients with persistently isolated HGPIN on the first follow-up biopsy had an immediate second 12-core biopsy when a raised PSA 
level or a suspicious digital rectal examination (DRE) finding or both was present, and those without raised PSA levels and/or abnormal DRE findings underwent the second 12-core biopsy at the end of the study at our department. Before each re-biopsy, patients underwent serum total PSA assays with RIA (Hybritech Inc.) and DRE. The PSA velocity during the year prior to diagnosis was determined from at least three serum PSA measurements between the first and the last measurements, and it was calculated as the linear regression slope of the PSA levels.

\section{Statistical analysis}

Student's $t$-test was used to compare the geometric means of serum EPCA absorbances between isolated HGPIN cases with and without subsequent cancer. The receiver operating characteristic (ROC) curves were constructed, and predictive accuracy of parameters for the presence of subsequent cancer was quantified by using the area under the curve (AUC). The differences of AUCs among parameters were assessed by the Mann-Whitney $U$ test. Univariate and multivariate Cox regression models were developed to evaluate the relative risk of subsequent cancer onset on follow-up. For all analyses, a $P$ value of $<0.05$ was considered to be statistically significant. The data analyses and interpretation were independently reviewed.

\section{Results}

The characteristics of isolated HGPIN patient are outlined in Table 1.

All eligible patients were followed up until one was subsequently diagnosed with PCa or until the study was terminated in June 2008. No patient was lost to follow-up. The median length of follow-up was 80.7 months, with a range of 60-100 months. In total, 31 patients were diagnosed with subsequent cancer on follow-up study, in which 19 cases were identified with a Gleason score of 6 , and the others were with a score of 7 . All 31 cancers were clinically localized tumors as determined by bone scan, computerized tomography of the abdomen and pelvis, chest radiography, and serum enzymatic prostatic acid phosphatase testing. All these patients had a radical prostatectomy as cancer treatment, and all had organ-confined pT2a-pT2c disease with negative surgical margins.

Our ELISA analysis of 112 individuals with isolated HGPIN showed that all had a detectable concentration of EPCA in the initial sera, with a mean of $0.64 \pm 0.13$ absorbance at $450 \mathrm{~nm}$. Figure 1 clearly showed that all
Table 1 Clinical characteristics of patients with isolated HGPIN

\begin{tabular}{|c|c|c|c|}
\hline Characteristics & $\begin{array}{l}\text { Eligible } \\
\text { patients } \\
(n=112)\end{array}$ & $\begin{array}{l}\text { All patients } \\
(n=143)\end{array}$ & $P$ value \\
\hline \multicolumn{4}{|l|}{ Age (years) } \\
\hline $\begin{array}{l}\text { Mean } \pm \text { S.D. } \\
\text { Weight }(\mathrm{kg})\end{array}$ & $64.6 \pm 1.0$ & $64.2 \pm 1.0$ & 0.37 \\
\hline $\begin{array}{l}\text { Mean } \pm \text { S.D. } \\
\text { Height }(\mathrm{cm})\end{array}$ & $67.3 \pm 5.2$ & $68.1 \pm 5.3$ & 0.34 \\
\hline Mean \pm s.D. & $169.4 \pm 4.4$ & $169.7 \pm 4.5$ & 0.41 \\
\hline \multicolumn{4}{|c|}{ Body mass indices (BMI, $\mathrm{kg} / \mathrm{m}^{2}$ ) } \\
\hline Mean \pm S.D. & $24.6 \pm 2.3$ & $24.4 \pm 2.3$ & 0.28 \\
\hline \multicolumn{4}{|c|}{ Prostate volume $(\mathrm{ml})$} \\
\hline Mean \pm s.D. & $43.3 \pm 8.2$ & $44.2 \pm 8.4$ & 0.30 \\
\hline \multicolumn{4}{|c|}{ Serum PSA (ng/ml) } \\
\hline $0-2.5$ & $64(57.1 \%)$ & $83(58 \%)$ & 0.11 \\
\hline $2.6-4.0$ & $29(25.9 \%)$ & $35(24.5 \%)$ & 0.17 \\
\hline $4.1-10.0$ & $15(13.4 \%)$ & $20(14 \%)$ & 0.16 \\
\hline$>10.0$ & $4(3.6 \%)$ & $5(3.5 \%)$ & 0.21 \\
\hline \multicolumn{4}{|l|}{ DRE findings } \\
\hline Normal & $94(83.9 \%)$ & $124(86.7 \%)$ & 0.14 \\
\hline Abnormal & $18(16.1 \%)$ & $19(13.3 \%)$ & 0.26 \\
\hline
\end{tabular}

PSA, prostate specific antigen; DRE, digital rectal examinations; HGPIN, high-grade prostatic intraepithelial neoplasia.

31 subsequent cancers had their initial serum EPCA levels $\geq 1.10$ absorbance. In addition, the ELISA also detected serum EPCA levels above 1.10 absorbance in 2 of 81 cases who were subsequently diagnosed without PCa. However, none of the individuals who had an initial serum EPCA level of $<1.10$ had

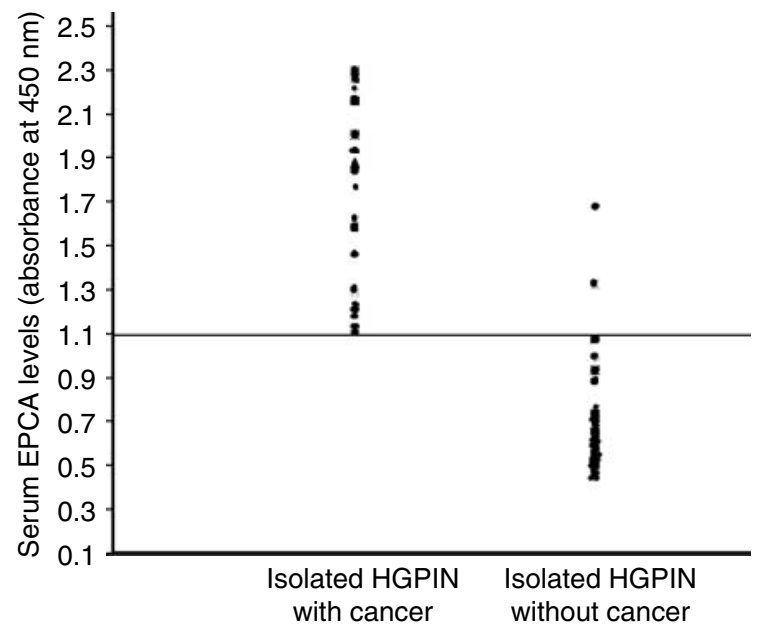

Figure 1 Serum analysis of EPCA. Initial serum EPCA levels were measured in 112 men with isolated HGPIN by using an indirect ELISA, of whom 31 were subsequently found with cancer and the others were not found with cancer on a follow-up of $\geq 5$ years. Each sample was done in triplicate and reported here as the mean representative of three independent experiments. 
Table 2 The specificity, sensitivity, PPV, and NPV of the parameters evaluated in the study

\begin{tabular}{|c|c|c|c|c|}
\hline Parameter & Specificity (\%) & Sensitivity (\%) & PPV (\%) & NPV $(\%)$ \\
\hline PSA velocity ( $>0.75 \mathrm{ng} / \mathrm{ml}$ per year) & 88.9 & 12.9 & 30.8 & 72.7 \\
\hline DRE findings (abnormal) & 80.2 & 6.5 & 11.1 & 69.1 \\
\hline Initial serum EPCA level $(\geq 1.10)$ & 97.5 & 100 & 93.9 & 100 \\
\hline
\end{tabular}

PSA, prostate specific antigen; DRE, digital rectal examinations; EPCA, early prostate cancer antigen; PPV, positive predictive values; NPV, negative predictive values.

progressed to clinically significant cancer during the follow-up period of 60-100 months. Thus, if a cut-off value of 1.10 was used, an overall specificity for this group with isolated HGPIN was $97.5 \%$, whereas the sensitivity for identifying subsequent malignancy was $100 \%$. The positive predictive values (PPV) and negative predictive values (NPV) were 93.9 and $100 \%$ respectively (Table 2). Intriguingly, the circulating EPCA level in the initial serum from patients with subsequent $\mathrm{PCa}$ was significantly higher than that from those without $(1.62 \pm 0.37$ vs $0.57 \pm 0.11, P<0.001)$. The post hoc analysis showed that men with isolated HGPIN who presented with an increased EPCA level in the initial serum $(\geq 1.10)$ were significantly more likely to develop a clinically significant cancer after the initial diagnosis than those who had an absorbance of $<1.10$ for serum EPCA $(P<0.001)$.

There is no statistically significant correlation between the Gleason scores and the EPCA levels in 31 isolated HGPIN men with cancer because all of the Gleason grade was either 6 or 7 ( $T$ test, $P=0.34$ ). Eighteen of 112 men had abnormal DRE findings noted at study entry, in which only two cancers were identified on the follow-up biopsies. Not surprisingly, DRE findings were not significantly associated with the subsequent development of cancer in isolated HGPIN patients $\left(\chi^{2}\right.$ test, $P=0.47$ ). Although 11 of 31 cases with subsequent cancer had the elevated PSA levels and the rest had a decline in PSA levels at the time to diagnosis when compared with their initial PSA concentrations, the elevated, declined, and overall PSA levels were all statistically insignificant (3.46 \pm 0.6 vs $2.78 \pm 0.3 \mathrm{ng} / \mathrm{ml}, P=0.11 ; 2.85 \pm 0.5$ vs $3.03 \pm 0.6 \mathrm{ng} / \mathrm{ml}, P=0.27$; and $3.16 \pm 0.6$ vs 2.91 $\pm 0.4 \mathrm{ng} / \mathrm{ml}, P=0.13$ respectively). In addition, PSA velocity was also found to be statistically insignificant between isolated HGPIN patients with and without subsequent cancer $(0.51 \mathrm{vs} 0.47 \mathrm{ng} / \mathrm{ml}$ per year, $P=0.18)$. Thirteen of 112 men had PSA velocity $>0.75 \mathrm{ng} / \mathrm{ml}$ per year, in which four cases were identified as having cancer on follow-up. Table 2 gives the calculated specificity, sensitivity, PPV, and NPV of PSA velocity $>0.75 \mathrm{ng} / \mathrm{ml}$ per year, and abnormal DRE findings and initial serum EPCA level respectively.
The ROC curves for PSA velocity, abnormal DRE findings, and initial serum EPCA level are plotted in Fig. 2, and the calculated AUCs and 95\% confidence intervals (CI) are presented in Table 3. Mann-Whitney $U$ test showed that the AUC was significantly greater for serum EPCA level than for PSA velocity and abnormal DRE findings $(P<0.001$ and $P<0.001$ respectively). Furthermore, univariate and multivariate Cox regression models were developed to evaluate the relative risk of subsequent cancer onset on follow-up. As shown in Table 4, the results confirmed that the increased serum EPCA levels $(\geq 1.10)$ carried a significantly higher risk of the presence of subsequent cancer in isolated HGPIN patients over a period of $\geq 5$ years after the initial diagnosis (relative risk, 3.32; 95\% CI, 2.62-5.03, $P<0.001$ ), whereas elevated serum PSA concentrations, PSA velocity, and abnormal DRE findings did not.

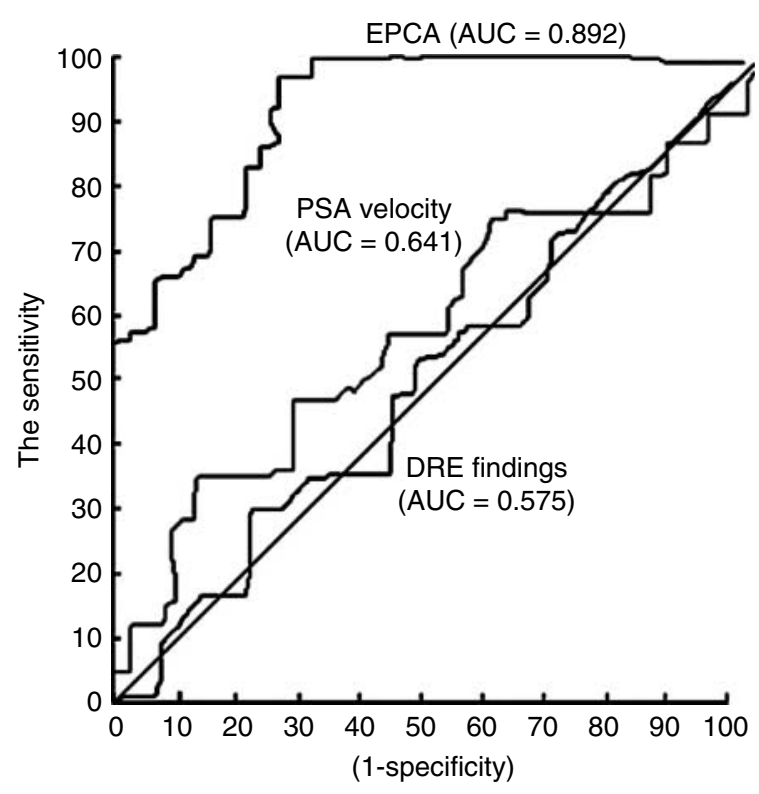

Figure 2 The receiver operating characteristic $(\mathrm{ROC})$ curves for PSA velocity, DRE findings, and initial serum EPCA level. AUC, area under ROC curve. 
Table 3 The AUC values and $95 \% \mathrm{Cl}$ calculated from ROC curves for the parameters evaluated in the study

\begin{tabular}{lcc}
\hline Parameter & AUC values & 95\% Cl \\
\hline PSA velocity & 0.641 & $0.583-0.762$ \\
Abnormal DRE findings & 0.575 & $0.417-0.664$ \\
Initial serum EPCA level & $0.892^{\star}$ & $0.753-0.968$ \\
\hline
\end{tabular}

PSA, prostate specific antigen; DRE, digital rectal examinations; EPCA, early prostate cancer antigen; AUC, area under the curve; $95 \% \mathrm{Cl}, 95 \%$ confidence intervals; ROC curves, receiver operating characteristic curves. *Statistically significant versus PSA velocity $(P<0.001)$ and abnormal DRE findings $(P<0.001)$ respectively, by the Mann-Whitney $U$ test.

\section{Discussion}

In the present study, we found a detectable circulating EPCA protein in the serum and a significant correlation between increased levels of serum EPCA and risk of subsequent cancer onset on follow-up in a group of individuals with isolated HGPIN. In a recent study by Paul et al. (2005), PIN alone appears not to be sufficient to result in detectable serum levels of EPCA. Our data are not in accordance with this finding. This discrepancy may be related to the different sensitivity of the assay to the populations of various radical/ethnic and geographic characteristics, such as Chinese and American. In addition, for blood-based tests using antibodies, the antibodies, especially polyclonal antibodies, can show even minor cross-reactivity with other antigens that circulate in the blood at various levels. Our follow-up studies revealed a statistically significant increase in serum EPCA levels in initial samples obtained from isolated HGPIN patients with subsequent $\mathrm{PCa}$ compared with samples obtained from those without subsequent PCa. Taken together with the previous immunostaining findings for EPCA
(Dhir et al. 2004, Uetsuki et al. 2005, Hansel et al. 2007), these data imply that EPCA may be used as a potential predictive marker for $\mathrm{PCa}$ onset. Moreover, the results presented here do not reveal a correlation between initial serum EPCA absorbance and Gleason score of subsequent tumor. This is in agreement with the results of Dhir et al. (2004), Paul et al. (2005), and Uetsuki et al. (2005), where tissue EPCA staining and plasma EPCA absorbances were not correlated with tumor stage in patients with $\mathrm{PCa}$, supporting the suggestion that EPCA expression occurs early during the development of cancer. In the present study, no statistically significant difference in regard to patients' age was found between men with subsequent cancer findings and those without subsequent cancer $(64.2 \pm 1.0$ vs $64.8 \pm 1.1, P=0.28)$, suggesting that age probably does not influence serum levels of EPCA in patients with isolated HGPIN.

Although serum PSA and its isoforms have been of continuous interest with regard to early detection and screening for PCa (Crawford \& Abrahamsson 2008, Schröder et al. 2008, Jansen et al. 2009), limitations in the sensitivity and specificity for the prediction of $\mathrm{PCa}$ are well understood (Welch et al. 2005, Steuber et al. 2008). Serum PSA level, PSA velocity, and abnormal DRE findings as parameters of clinical importance have more recently been evaluated to predict the presence of PCa among men with HGPIN; however, the results were still conflict (Cheng et al. 2004, Moore et al. 2005, Herawi et al. 2006, Akhavan et al. 2007, Loeb et al. 2007). In this study, serum PSA changes, PSA velocity, and abnormal DRE findings were not shown to be helpful in predicting the likelihood of cancer on the repeat biopsy in patients with isolated HGPIN. This is in agreement with the results of other authors (Cheng et al. 2004, Moore et al. 2005,

Table 4 Univariate and multivariate Cox regression analyses for relative risk of the subsequent prostate cancer (PCa) onset in isolated HGPIN

\begin{tabular}{|c|c|c|c|}
\hline Covariate & Relative risk & $95 \% \mathrm{Cl}$ & $P$ value \\
\hline \multicolumn{4}{|l|}{ Univariate } \\
\hline Increased serum EPCA level ( $\geq 1.10$ absorbance) & 6.84 & $5.34-8.76$ & $<0.001$ \\
\hline Elevated serum PSA level & 0.72 & $0.45-1.61$ & 0.52 \\
\hline PSA velocity ( $>0.75 \mathrm{ng} / \mathrm{ml}$ per year) & 1.16 & $0.77-2.18$ & 0.24 \\
\hline Abnormal DRE findings & 0.45 & $0.26-1.15$ & 0.86 \\
\hline \multicolumn{4}{|l|}{ Multivariate } \\
\hline Increased serum EPCA level ( $\geq 1.10$ absorbance) & 3.32 & $2.62-5.03$ & $<0.001$ \\
\hline Elevated serum PSA level & 0.41 & $0.27-1.11$ & 0.63 \\
\hline PSA velocity ( $>0.75 \mathrm{ng} / \mathrm{ml}$ per year) & 0.75 & $0.48-1.24$ & 0.18 \\
\hline Abnormal DRE findings & 0.78 & $0.16-0.75$ & 0.88 \\
\hline
\end{tabular}

PSA, prostate specific antigen; DRE, digital rectal examinations; EPCA, early prostate cancer antigen; HGPIN, high-grade prostatic intraepithelial neoplasia; $95 \% \mathrm{Cl}, 95 \%$ confidence intervals. 
Herawi et al. 2006, Akhavan et al. 2007), and our recent findings (Zhao \& Shen 2007). Henshall et al. (2001) reported that overexpression of the cell cycle inhibitor p16INK4A in HGPIN is an independent predictor of $\mathrm{PCa}$ relapse after radical prostatectomy, which is the first prognostic marker identified in patients with HGPIN. Microvessel density as a molecular marker was found to distinguish HGPIN that has the potential of progressing to PCa from those that do not have (Sinha et al. 2004). Our recent studies revealed, using in situ hybridization analysis, that prostate stem cell antigen expression in HGPIN tissues on the initial biopsy of prostate was independently predictive of the onset of subsequent cancer over $\geq 5$ years of follow-up in a group of 66 men with isolated HGPIN (odd ratio, 3.23; 95\% CI: 1.57-6.15, $P<0.001$; Zhao \& Shen 2007). Most recently, a prospective study showed that the prostate cancer gene 3 (PCA3) score was significantly higher in the urine of men with HGPIN versus those without HGPIN, suggesting the potential of PCA3 in identifying men at risk of developing PCa (Haese et al. 2008). However, the identification of novel biomarkers in blood is still needed.

With the observation that isolated HGPIN patients had the detectable circulating EPCA protein, another concern to us in this study is whether blood levels of EPCA can predict the progression to clinical cancer in these individuals. Thus, we evaluated the relationship of initial serum EPCA levels and the subsequent PCa risk over a follow-up period of $\geq 5$ years in 112 cases with isolated HGPIN. The results presented here first demonstrated a significant trend for those who had an increased initial serum level of EPCA $(\geq 1.10$ absorbance) to progress to a clinically significant cancer after the initial diagnosis of isolated HGPIN. Cancer diagnostic tests must be both specific and sensitive. Our ELISA showed that initial serum EPCA absorbance $\geq 1.10$ does not only exhibit an overall specificity of $97.5 \%$ and a sensitivity of $100 \%$ for detection of subsequent cancer but also has a PPV of 93.9\% and a NPV of $100 \%$ in this set of patients. Our results are similar to those of Paul et al. (2005) and Leman et al. (2007, 2009), where the blood-based ELISA for both EPCA and EPCA-2 showed high specificity and sensitivity for patients with PCa. Taken together, these data support the potential of using the EPCA ELISA to identify men with isolated HGPIN who do have carcinoma subsequently. The estimation of AUC values from ROC curves for initial serum EPCA level, PSA velocity, and abnormal DRE findings showed that initial serum EPCA level had the largest AUC among those parameters, demonstrating the most promising marker for predicting the presence of subsequent cancer in isolated HGPIN patients. Furthermore, univariate and multivariate Cox regression analyses confirmed that initial serum EPCA level $\geq 1.10$ carried a significantly increased risk for the development of subsequent $\mathrm{PCa}$ in the process of isolated HGPIN. Taken together, our findings presented here substantiate the possible role of EPCA as a serum marker for identifying men with isolated HGPIN who are at higher risk to subsequently develop cancers early in the disease process, even at an earlier interval than 5 years. Although a larger study is required for definitive conclusions and we are now in the process of setting up such a study, the preliminary results of this study may have significant clinical implications for managing a patient with isolated HGPIN. The individuals with isolated HGPIN who had initial serum EPCA absorbances $\geq 1.10$ absorbance would be followed up more closely than those who had initial serum EPCA levels below 1.10, regardless of PSA changes and DRE findings.

In conclusion, this preliminary study, to the best of our knowledge, first demonstrated a detectable level of EPCA in the initial serum of men with isolated HGPIN by an ELISA and a statistically significant difference of initial serum EPCA concentrations between isolated HGPIN individuals with subsequent $\mathrm{PCa}$ and those without subsequent PCa. The findings that those individuals with increased serum levels of EPCA ( $\geq 1.10$ absorbance) are at higher risk to subsequently develop clinical cancer over a period of $\geq 5$ years make EPCA potentially a very powerful serum biomarker for the early detection of $\mathrm{PCa}$ in patients with isolated HGPIN.

\section{Declaration of interest}

The authors declare that there is no conflict of interest that could be perceived as prejudicing the impartiality of the research reported.

\section{Funding}

This work was supported by the grant from Science \& Technology Planning Project of Guangdong Province, China (No. 2008B080701035).

\section{References}

Abdel-Khalek M, El-Baz M \& Ibrahiem el-H 2004 Predictors of prostate cancer on extended biopsy in patients with high grade prostatic intraepithelial neoplasia: a multivariate analysis model. British Journal of Urology International 94 528-533. 
Akhavan A, Keith JD, Bastacky SI, Cai C, Wang Y \& Nelson JB 2007 The proportion of cores with highgrade prostatic intraepithelial neoplasia on extendedpattern needle biopsy is significantly associated with prostate cancer on site-directed repeat biopsy. British Journal of Urology International 99 765-769.

Ayala AG \& Ro JY 2007 Prostatic intraepithelial neoplasia: recent advances. Archives of Pathology \& Laboratory Medicine 131 1257-1266.

Cheng L, Paterson RF, Beck SD \& Parks J 2004 Prostatic intraepithelial neoplasia: an update. Clinical Prostate Cancer 3 26-30.

Chin AI, Dave DS \& Rajfer J 2007 Is repeat biopsy for isolated high-grade prostatic intraepithelial neoplasia necessary? Reviews in Urology 9 124-131.

Crawford ED \& Abrahamsson PA 2008 PSA-based screening for prostate cancer: how does it compare with other cancer screening tests? European Urology 54 262-273.

Dhir R, Vietmeier B, Arlotti J, Acquafondata M, Landsittel D, Masterson R \& Getzenberg RH 2004 Early identification of individuals with prostate cancer in negative biopsies. Journal of Urology 171 1419-1423.

Dovey Z, Corbishley CM \& Kirby RS 2005 Prostatic intraepithelial neoplasia: a risk factor for prostate cancer. Canadian Journal of Urology 12 (Supplement 1) 49-52.

Freeman AA 2006 Precursor lesions of prostatic adenocarcinoma. In Prostate Cancer Principles and Practice, edn 1, pp 122. Eds RS Kirby, AW Partin, M Feneley \& JK Parsons. London, UK: Taylor \& Francis.

Godoy G \& Taneja SS 2008 Contemporary clinical management of isolated high-grade prostatic intraepithelial neoplasia. Prostate Cancer and Prostatic Diseases 11 20-31.

Gokden N, Roehl KA, Catalona WJ \& Humphrey PA 2005 High-grade prostatic intraepithelial neoplasia in needle biopsy as risk factor for detection of adenocarcinoma: current level of risk in screening population. Urology 65 538-542.

Haese A, de la Taille A, van Poppel H, Marberger M, Stenzl A, Mulders PF, Huland H, Abbou CC, Remzi M, Tinzl M et al. 2008 Clinical utility of PCA3 urine assay in European men scheduled for repeat biopsy. European Urology 54 1081-1088.

Hansel DE, DeMarzo AM, Platz EA, Jadallah S, Hicks J, Epstein JI, Partin AW \& Netto GJ 2007 Early prostate cancer antigen expression in predicting presence of prostate cancer in men with histologically negative biopsies. Journal of Urology 177 1736-1740.

Henshall SM, Quinn DI, Lee CS, Head DR, Golovsky D, Brenner PC, Delprado W, Stricker PD, Grygiel JJ \& Sutherland RL 2001 Overexpression of the cell cycle inhibitor p16INK4A in highgrade prostatic intraepithelial neoplasia predicts early relapse in prostate cancer patients. Clinical Cancer Research 7 544-550.

Herawi M, Kahane H, Cavallo C \& Epstein JI 2006 Risk of prostate cancer on first re-biopsy within 1 year following a diagnosis of high grade prostatic intraepithelial neoplasia is related to the number of cores sampled. Journal of Urology 175 121-124.

Jansen FH, Roobol M, Jenster G, Schröder FH \& Bangma CH 2009 Screening for prostate cancer in 2008 II: the importance of molecular subforms of prostate-specific antigen and tissue kallikreins. European Urology 55 $563-574$.

Joniau S, Goeman L, Pennings J \& Van Poppel H 2005 Prostatic intraepithelial neoplasia (PIN): importance and clinical management. European Urology 48 379-385.

Leman ES, Cannon GW, Trock BJ, Sokoll LJ, Chan DW, Mangold L, Partin AW \& Getzenberg RH 2007 EPCA-2: a highly specific serum marker for prostate cancer. Urology 69 714-720.

Leman ES, Magheli A, Cannon GW, Mangold L, Partin AW \& Getzenberg RH 2009 Analysis of a serum test for prostate cancer that detects a second epitope of EPCA-2. Prostate 69 1188-1194.

Loeb S, Roehl KA, Yu X, Han M \& Catalona WJ 2007 Use of prostate specific antigen velocity to follow up patients with isolated high-grade prostatic intraepithelial neoplasia on prostate biopsy. Urology 69 108-112.

Montironi R, Mazzucchelli R, Lopez-Beltran A, Cheng L \& Scarpelli M 2007 Mechanisms of disease: high-grade prostatic intraepithelial neoplasia and other proposed preneoplastic lesions in the prostate. Nature Clinical Practice. Urology 4 321-332.

Moore CK, Karikehalli S, Nazeer T, Fisher HA, Kaufman RP Jr \& Mian BM 2005 Prognostic significance of high grade prostatic intraepithelial neoplasia and atypical small acinar proliferation in the contemporary era. Journal of Urology 173 70-72.

Paul B, Dhir R, Landsittel D, Hitchens MR \& Getzenberg RH 2005 Detection of prostate cancer with a blood-based assay for early prostate cancer antigen. Cancer Research 65 4097-4100.

Roscigno M, Scattoni V, Freschi M, Raber M, Colombo R, Bertini R, Montorsi F \& Rigatti P 2004 Monofocal and plurifocal high-grade porstatic intraepithelial neoplasia on extended prostate biopsies: factors predicting cancer detection on extended repeat biopsy. Urology 63 1105-1110.

Schröder FH, Carter HB, Wolters T, van den Bergh RC, Gosselaar C, Bangma CH \& Roobol MJ 2008 Early detection of prostate cancer in 2007. Part 1: PSA and PSA kinetics. European Urology 53 468-477.

Singh H, Canto EI, Shariat SF, Kadmon D, Miles BJ, Wheeler TM \& Slawin KM 2004 Predictors of prostate cancer after initial negative systematic 12 core biopsy. Journal of Urology 171 1850-1854.

Sinha AA, Quast BJ, Reddy PK, Lall V, Wilson MJ, Qian J \& Bostwick DG 2004 Microvessel density as a molecular marker for identifying high-grade prostatic intraepithelial neoplasia precursors to prostate cancer. Experimental and Molecular Pathology 77 153-159. 
Steuber T, O’Brien MF \& Lilja H 2008 Serum markers for prostate cancer: a rational approach to the literature. European Urology 54 31-40.

Uetsuki H, Tsunemori H, Taoka R, Haba R, Ishikawa M \& Kakehi Y 2005 Expression of a novel biomarker, EPCA, in adenocarcinomas and precancerous lesions in the prostate. Journal of Urology 174 514-518.
Welch HG, Schwartz LM \& Woloshin S 2005 Prostatespecific antigen levels in the United States: implications of various definitions for abnormal. Journal of the National Cancer Institute 97 1132-1137.

Zhao ZG \& Shen WL 2007 Prostate stem cell antigen (PSCA) mRNA expression in prostatic intraepithelial neoplasia: implications for the development of prostate cancer. Prostate 67 1143-1151. 